\title{
Conscious Sedation versus General Anesthesia during Endovascular Acute Ischemic Stroke Treatment: A Systematic Review and Meta-Analysis
}

\author{
W. Brinjikji, M.H. Murad, A.A. Rabinstein, H.J. Cloft, G. Lanzino, and D.F. Kallmes
}

\begin{abstract}
BACKGROUND AND PURPOSE: A number of studies have suggested that anesthesia type (conscious sedation versus general anesthesia) during intra-arterial treatment for acute ischemic stroke has implications for patient outcomes. We performed a systematic review and meta-analysis of studies comparing the clinical and angiographic outcomes of the 2 anesthesia types.
\end{abstract}

MATERIALS AND METHODS: In March 2014, we conducted a computerized search of MEDLINE and EMBASE for reports on anesthesia and endovascular treatment of acute ischemic stroke. Using random-effects meta-analysis, we evaluated the following outcomes: recanalization rate, good functional outcome (mRS $\leq 2$ ), asymptomatic and symptomatic intracranial hemorrhage, death, vascular complications, respiratory complications, procedure time, time to groin, and time from symptom onset to recanalization.

RESULTS: Nine studies enrolling 1956 patients (814 with general anesthesia and 1142 with conscious sedation) were included. Compared with patients treated by using conscious sedation during stroke intervention, patients undergoing general anesthesia had higher odds of death $(\mathrm{OR}=2.59 ; 95 \% \mathrm{Cl}, 1.87-3.58)$ and respiratory complications $(\mathrm{OR}=2.09 ; 95 \% \mathrm{Cl}, 1.36-3.23)$ and lower odds of good functional outcome $(\mathrm{OR}=0.43 ; 95 \% \mathrm{Cl}, 0.35-0.53)$ and successful angiographic outcome $(\mathrm{OR}=0.54 ; 95 \% \mathrm{Cl}, 0.37-0.80)$. No difference in procedure time $(P=.28)$ was seen between the groups. Preintervention NIHSS scores were available from 6 studies; in those, patients receiving general anesthesia had a higher average NIHSS score.

CONCLUSIONS: Patients with acute ischemic stroke undergoing intra-arterial therapy may have worse outcomes with general anesthesia compared with conscious sedation. However, the difference in stroke severity at the onset may confound the comparison in the available studies; thus, a randomized trial is necessary to confirm this association.

ABBREVIATION: ICH = intracranial hemorrhage

ntra-arterial recanalization for acute ischemic stroke is commonly used in patients with large-vessel occlusion. ${ }^{1}$ Timely recanalization of the occluded vessel with either IV-tPA or intraarterial therapy is essential in preventing neuronal death and improving patient outcome. ${ }^{2}$ A number of factors affect patient outcomes following endovascular recanalization, possibly including choice of anesthetic agent during the procedure. Moderate conscious sedation and general anesthesia with intubation are the

\footnotetext{
Received June 11, 2014; accepted after revision September 6.

From the Departments of Radiology (W.B., H.J.C., G.L., D.F.K.), Neurology (A.A.R.), and Neurosurgery (H.J.C., G.L., D.F.K.), and Center for the Science of Healthcare Delivery and the Division of Preventive Medicine (M.H.M.), Mayo Clinic, Rochester, Minnesota.

Please address correspondence to Waleed Brinjikji, MD, 200 1st St SW, Rochester, MN 55905; e-mail: brinjikji.waleed@mayo.edu

Indicates article with supplemental on-line photo.

Evidence-Based Medicine Level 1.

http://dx.doi.org/10.3174/ajnr.A4159
}

2 most commonly used anesthesia techniques for patients with acute ischemic stroke undergoing endovascular recanalization. ${ }^{3}$ General anesthesia is often the preferred method due to the perceptions of improved procedural safety and efficacy. ${ }^{3}$ However, conscious sedation and local anesthesia allow operators to monitor neurologic status during the procedure and avoid delays in procedure initiation. ${ }^{4}$ Furthermore, conscious sedation may be associated with improved hemodynamic stability compared with general anesthesia. Due to the continuing debate regarding anesthesia choices during intra-arterial treatment of acute ischemic stroke, we performed a meta-analysis of studies comparing outcomes of patients with stroke receiving general anesthesia and conscious sedation during the procedures. ${ }^{5,6}$

\section{MATERIALS AND METHODS Literature Search}

To identify comparative studies on general anesthesia versus conscious sedation during endovascular treatment of acute ischemic stroke, we searched 3 databases from 1990 to March 2014: Ovid 
MEDLINE, Ovid EMBASE, and the Web of Science. Ovid MEDLINE and Ovid EMBASE use controlled vocabulary. The Web of Science is text word-based but tends to be more current and multidisciplinary. The initial search terms were conscious sedation, general anesthesia, and intracranial embolism and thrombosis or stroke. These were combined with treatment techniques: endovascular, fibrinolytic agents, thromboembolism, catheter, transcatheter, thrombolysis, fibrinolysis, recanalization, embolectomy, or thrombectomy (subject heading available in EMBASE, but not MEDLINE). We also searched references from multiple articles to find any additional studies on anesthesia and outcomes of endovascular treatment of acute ischemic stroke not found in the initial literature search.

Identified studies from the literature search were then further evaluated for inclusion in the meta-analysis. Inclusion criteria were the following: 1) studies comparing outcomes of the 2 groups: general anesthesia and conscious sedation/local anesthesia; 2) studies reporting separate angiographic and clinical outcomes for the general anesthesia and conscious sedation/local anesthesia groups. Exclusion criteria were the following: 1) case reports, 2) studies not separating outcomes by anesthesia type, 3 ) noncomparative studies (ie, studies with only 1 group: general anesthesia or conscious sedation). Two authors reviewed the articles for inclusion. Following selection of the articles, the data were abstracted by 1 author.

\section{Quality Assessment}

Quality assessment of the studies was performed by using the Newcastle-Ottawa Scale. This tool is used for assessing the quality of nonrandomized studies included in systematic reviews and/or meta-analyses. Each study is judged on 8 items categorized into 3 groups: 1) selection of the study groups, 2) comparability of the study groups, and 3) ascertainment of the outcome of interest. One star is awarded for each quality item; 4 stars are the maximum for the selection of groups, 2 stars are the maximum for comparability, and 3 stars are the maximum for ascertainment of outcome. The highest quality studies are awarded up to 9 stars. ${ }^{7}$

\section{Outcome Variables}

Good functional outcome, defined as a modified Rankin Scale score of $\leq 2$ at 90 days following endovascular treatment, was the primary end point of this study. Other studied outcomes included mortality, successful recanalization/angiographic outcome (Thrombolysis in Myocardial Infarction $\geq 2$ or TICI 2b/3), asymptomatic intracranial hemorrhage (ICH), symptomatic $\mathrm{ICH}$, other vascular complications including dissections and vessel perforations, respiratory complications including respiratory failure and pneumonia, procedure time, time from symptom onset to revascularization, and time from arrival at the hospital to groin puncture.

\section{Statistics}

From each study, we extracted a $2 \times 2$ table for binary outcomes, the mean group sample size, and a measure of variability for continuous outcomes. Random-effects meta-analysis was used for pooling across studies. ${ }^{8}$ The $\mathrm{I}^{2}$ statistic was used to express the proportion of inconsistency that was not attributable to chance. ${ }^{9}$
Meta-analysis results were expressed as odds ratio for binary outcomes and weighted mean difference for continuous outcomes with respective $95 \%$ confidence intervals. When assessing continuous outcomes, some studies reported mean values with corresponding SDs, while others reported median values with interquartile ranges. If a median and interquartile range were reported, these were converted to a mean and SD on the basis of the assumption of a log-normal distribution of the original measure. We planned to explore the impact of publication bias by constructing funnel plots and testing their symmetry if a sufficient number of studies $(>20)$ were available. Meta-analysis was conducted by using Comprehensive Meta-Analysis, Version 2.2 (Comprehensive Meta-Analysis, Englewood, New Jersey).

Because the study subjects were not randomized by anesthesia type, we were concerned about a lack of similarity between the 2 study groups (ie, patients with worse prognosis receiving 1 of the 2 interventions). Therefore, we planned to conduct metaregression, in which the dependent outcome was the effect size (log of the OR of the primary outcome, the odds of good neurologic outcome), and the explanatory variables (independent variables) were initial stroke severity categorized by the baseline average NIHSS score and the type of anesthesia (general anesthesia versus conscious sedation). Studies were weighted in metaregression by using their precision. The results of the metaregression were presented as an odds ratio adjusted for NIHSS score. Metaregression was conducted by using STATA, Version 12.1 (StataCorp, College Station, Texas). We also performed a sensitivity analysis examining the comparative outcomes, including only those studies that reported baseline NIHSS scores.

\section{RESULTS}

\section{Literature Search}

One hundred twenty-three articles were found on the initial literature search. Of these, 102 (82.9\%) were excluded after reading the abstracts alone because they were not found to be relevant to our study. Of the remaining 21 articles, 3 (2.4\%) were excluded because they mixed outcomes of endovascular stroke treatment with other endovascular procedures (ie, stent placement, aneurysm coiling, and so forth); 5 articles (4.1\%) were excluded because they only included 1 treatment group; and 4 articles (3.3\%) were excluded because they were review articles. In total, 9 articles (7.3\%) with 1956 patients (814 with general anesthesia and 1142 with conscious sedation) were included in this study. No studies randomized patients to general anesthesia or conscious sedation. The largest study had 1079 patients (428 with general anesthesia and 651 with conscious sedation), and the smallest study had 66 patients ( 9 with general anesthesia and 57 with conscious sedation). All studies had at least 7 stars on the Newcastle-Ottawa scale. A summary of included studies is provided in Table 1.

\section{Outcomes}

General anesthesia was associated with a lower odds of favorable functional outcome (ie, mRS $\leq 2$ ) compared with conscious sedation $(\mathrm{OR}=0.43 ; 95 \% \mathrm{CI}, 0.35-0.53 ; P<.01)$ (Figure). General anesthesia was also associated with a lower odds of successful recanalization $(\mathrm{OR}=0.54 ; 95 \% \mathrm{CI}, 0.37-0.80 ; P<.01)$. Patients 
Table 1: Studies included in the meta-analysis

\begin{tabular}{|c|c|c|c|c|c|c|}
\hline Authors, Year & No. with GA & No. with CS & Type of Endovascular Treatment & Selection & Comparability & Outcome \\
\hline Abou-Chebl et al, $2014^{28}$ & 196 & 85 & Solitaire stent ${ }^{a}$ & $\star \star \star \star \star^{\mathrm{b}}$ & & $\star \star \star \star$ \\
\hline Davis et al, $2012^{12}$ & 48 & 48 & IA tPA and mechanical thrombectomy & $\star \star \star \star \star$ & $\star$ & $\star \star \star$ \\
\hline Hassan et al, $2012^{21}$ & 53 & 83 & Endovascular technique not specified & 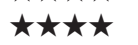 & & 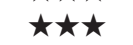 \\
\hline Jumaa et al, $2010^{17}$ & 53 & 73 & IA tPA and mechanical thrombectomy & $\star \star \star \star \star ~$ & $\star$ & $\star \star \star$ \\
\hline Langner et al, $2013^{26}$ & 19 & 105 & Mechanical thrombectomy & $\star \star \star \star \star$ & $\star$ & $\star \star \star \star$ \\
\hline Li et al, $2014^{25}$ & 35 & 74 & Mechanical thrombectomy, IA tPA & 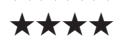 & $\star$ & 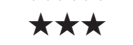 \\
\hline Nichols et al, $2010^{27}$ & 26 & 49 & IA tPA, low-energy ultrasound & 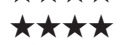 & $\star$ & 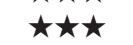 \\
\hline Sugg et al, $2010^{30}$ & 9 & 57 & Mechanical thrombectomy & $\star \star \star \star$ & $\star$ & $\star \star \star$ \\
\hline Abou-Chebl et al, $2010^{16}$ & 428 & 651 & IA tPA, mechanical thrombectomy, stent & $\star \star \star \star \star ~$ & $\star$ & $\star \star \star$ \\
\hline
\end{tabular}

Note:-GA indicates general anesthesia; CS, conscious sedation; IA, intra-arterial.

${ }^{a}$ Covidien, Irvine, California.

${ }^{b}$ One star is awarded for each quality item; 4 stars are the maximum for selection of groups, 2 stars are the maximum for comparability, and 3 stars are the maximum for ascertainment of outcome.

\begin{tabular}{|c|c|c|c|c|c|c|c|c|}
\hline \multirow[t]{2}{*}{$\underline{\text { Study name }}$} & \multicolumn{4}{|c|}{$\underline{\text { Statistics for each study }}$} & & \multicolumn{3}{|c|}{ Odds ratio and $95 \% \mathrm{Cl}$} \\
\hline & $\begin{array}{l}\text { Odds } \\
\text { ratio }\end{array}$ & $\begin{array}{c}\text { Lower } \\
\text { limit }\end{array}$ & $\begin{array}{l}\text { Upper } \\
\text { limit }\end{array}$ & $\mathrm{p}$-Value & & & & \\
\hline Sugg et al, AJNR, 2010 & 0.12 & 0.01 & 1.03 & 0.05 & & & & \\
\hline Davis et al, Anesthesiology, 2012 & 0.11 & 0.04 & 0.30 & 0.00 & & & & \\
\hline Abou-Chebl et al, Stroke, 2010 & 0.52 & 0.40 & 0.68 & 0.00 & & & & \\
\hline Abou-Chebl et al, Stroke, 2014 & 0.49 & 0.29 & 0.83 & 0.01 & & & & \\
\hline Li et al, J Neurosurg Anesthesiol, 2014 & 0.74 & 0.22 & 2.51 & 0.63 & & & & \\
\hline Jumaa et al, Stroke, 2010 & 0.35 & 0.16 & 0.78 & 0.01 & & & & \\
\hline Nichols et al, JNIS, 2010 & 0.19 & 0.06 & 0.56 & 0.00 & & & & \\
\hline \multirow[t]{3}{*}{ Hassan et al, Neurocrit Care, 2012} & 0.25 & 0.11 & 0.59 & 0.00 & & & & \\
\hline & 0.43 & 0.35 & 0.53 & 0.00 & & & & \\
\hline & & & & & 0.01 & 0.1 & 10 & 100 \\
\hline
\end{tabular}

FIG 1. Forest plot of meta-analysis results for good functional outcome (mRS $\leq 2)$.

\section{Study Heterogeneity}

$\mathrm{I}^{2}$ values were $<50 \%$ for the following outcomes: asymptomatic $\mathrm{ICH}\left(\mathrm{I}^{2}=\right.$ $41 \%)$, death $\left(\mathrm{I}^{2}=0 \%\right)$, other vascular complications $\left(\mathrm{I}^{2}=8 \%\right)$, recanalization success $\left(\mathrm{I}^{2}=11 \%\right)$, respiratory complications $\left(\mathrm{I}^{2}=0 \%\right)$, and symptomatic ICH $\left(\mathrm{I}^{2}=0 \%\right) . \mathrm{I}^{2}$ values were $>50 \%$ (indicating substantial heterogeneity) for the following outcomes: $\mathrm{mRS} \leq 2$ $\left(\mathrm{I}^{2}=55 \%\right)$, time to revascularization $\left(\mathrm{I}^{2}=\right.$ $60 \%)$, time to groin puncture $\left(\mathrm{I}^{2}=83 \%\right)$, and procedure time $\left(\mathrm{I}^{2}=91 \%\right)$.
Table 2: Meta-analysis results: categoric outcomes

\begin{tabular}{lccc}
\hline & OR GA vs CS & $95 \% \mathbf{C l}$ & $\boldsymbol{P}$ Value \\
\hline Death & 2.59 & $1.87-3.58$ & $<.01$ \\
Good functional outcome $^{\mathrm{a}}$ & 0.43 & $0.35-0.53$ & $<.01$ \\
Successful recanalization & 0.49 & $0.33-0.72$ & $<.01$ \\
sICH & 1.34 & $0.95-1.87$ & .09 \\
alCH & 1.24 & $0.94-1.62$ & .12 \\
Other vascular complications & 1.22 & $0.68-2.18$ & .5 \\
Respiratory complications & 2.09 & $1.36-3.23$ & $<.01$ \\
\hline
\end{tabular}

Note:- alCH indicates asymptomatic intracranial hemorrhage; $\mathrm{slCH}$, symptomatic intracranial hemorrhage.

${ }^{\text {a }}$ Modified Rankin scale score of $\leq 2$.

receiving general anesthesia had higher odds of mortality $(\mathrm{OR}=$ 2.59; 95\% CI, 1.87-3.58; $P<.01)$ and respiratory complications $(\mathrm{OR}=2.09 ; 95 \% \mathrm{CI}, 1.36-3.23 ; P<.01)$. There was no significant difference in the odds of asymptomatic ICH $(\mathrm{OR}=1.24$; 95\% CI, 0.94-1.62; $P=.12$ ), symptomatic ICH (OR $=1.34 ; 95 \%$ CI, $0.95-1.87 ; P=.09$ ), or other vascular complications (OR = $1.22 ; 95 \% \mathrm{CI}, 0.68-2.18 ; P=.50)$ between groups. These findings are summarized in Table 2. Forest plots for other measures are included in On-line Figs 1-6.

Mean time to groin was 136 minutes 20 seconds \pm 54 minutes for general anesthesia compared with 117 minutes 20 seconds \pm 56 minutes 20 seconds for conscious sedation $(P=.24)$. Mean procedure time was 104 minutes 20 seconds for general anesthesia compared with 89 minutes \pm 44 seconds for conscious sedation $(P=.28)$. Mean time from symptom onset to revascularization was $329 \mathrm{~min}-$ utes 43 seconds \pm 173 minutes for general anesthesia compared with 354 minutes 51 seconds \pm 265 minutes for conscious sedation $(P=$ $.17)$.

\section{Metaregression and Publication Bias}

Adjusting for NIHSS score by using metaregression for the main outcome (odds of having good functional outcomes) yielded an odds ratio of 0.38 ; which was similar to the unadjusted estimate of 0.43; however, the $95 \%$ CI became statistically insignificant $(0.12$, 1.22). The number of included studies in this analysis (with an available NIHSS score) was only 6; therefore, this adjustment is not reliable and likely underpowered.

Evaluation of publication bias was not possible due to the small number of included studies.

\section{Sensitivity Analysis}

Outcomes from our sensitivity analysis, including only those studies that reported baseline NIHSS scores, are provided in Table 3. Similar to the results in the overall analysis, in the sensitivity analysis, general anesthesia was associated with higher odds of death $(\mathrm{OR}=2.22 ; 95 \% \mathrm{CI}, 1.54-3.20)$ and respiratory complications $(\mathrm{OR}=2.03 ; 95 \% \mathrm{CI}, 1.12-3.68)$. General anesthesia was associated with lower odds of recanalization ( $\mathrm{OR}=0.48 ; 95 \%$ CI, $0.30-0.76)$ and lower odds of good neurologic outcome $(\mathrm{OR}=0.38 ; 95 \% \mathrm{CI}, 0.25-0.60)$. No differences in procedure time $(P=.69)$, time to groin $(P=.77)$, or time to revascularization $(P=1.00)$ were seen.

\section{DISCUSSION}

Our meta-analysis demonstrated that patients receiving conscious sedation had higher rates of good functional outcome and recanalization and decreased rates of mortality and respiratory complications compared with those receiving general anesthesia. No difference in procedure times, time to recanalization, or time 
Table 3: Sensitivity analysis excluding studies without baseline NIHSS data: categoric outcomes

\begin{tabular}{lccccc}
\hline & $\begin{array}{c}\text { No. of } \\
\text { Studies }\end{array}$ & $\begin{array}{c}\text { OR GA } \\
\text { vs CS }\end{array}$ & $\begin{array}{c}95 \% \mathrm{Cl} \\
\text { Value }\end{array}$ & $\mathbf{I}^{\mathbf{2}}$ \\
\hline alCH & 2 & 1.17 & $0.89-1.54$ & .27 & $\mathrm{NA}$ \\
Death & 5 & 2.22 & $1.54-3.20$ & .00 & 0 \\
mRS $\leq 2$ & 6 & 0.38 & $0.25-0.60$ & .00 & 56 \\
Other vasc comp & 3 & 1.66 & $0.56-4.93$ & .36 & 37 \\
Recan (TIMI $\geq 2)$ & 4 & 0.55 & $0.35-0.87$ & .01 & 0 \\
Resp comp & 3 & 2.03 & $1.12-3.68$ & .02 & 0 \\
sICH & 5 & 1.21 & $0.83-1.75$ & .32 & 0 \\
\hline
\end{tabular}

Note:-NA indicates not applicable; vasc comp, vascular complications; Recan, recanalization; Resp comp, respiratory complications; TIMI, Thrombolysis in Myocardial Infarction; GA, general anesthesia; $\mathrm{CS}$, conscious sedation; alCH, asymptomatic intracranial hemorrhage; $\mathrm{sICH}$, symptomatic intracranial hemorrhage.

to groin was seen between groups. Rates of $\mathrm{ICH}$ and vascular complications were similar between the 2 groups as well. Following adjustment for the baseline NIHSS score, general anesthesia was associated with lower, but not statistically significant, odds of good functional outcome. Because only 6 of the 9 studies in this meta-analysis included information on the baseline NIHSS score, the lack of significance of the association in the adjusted analysis may have been related to the decreased statistical power. Given the superior outcomes of patients receiving conscious sedation compared with those receiving general anesthesia, these data suggest that conscious sedation should generally be the anesthesia technique of choice during endovascular recanalization for treatment of acute ischemic stroke.

A number of factors likely contributed to the higher morbidity and mortality rates seen in the general anesthesia group. Inhalational anesthetic agents are associated with a higher risk of cerebral hypoperfusion and increased ischemic injury. ${ }^{10,11}$ Davis et $\mathrm{al}^{12}$ found that patients with ischemic stroke receiving endovascular recanalization had higher rates of intraprocedural hypotension with general anesthesia than with conscious sedation. Inhalational anesthetic agents, such as isoflurane, have been found to steal flow from ischemic areas with poor autoregulation by inducing cerebral vasodilation. ${ }^{10,13}$ Induction and recovery phases of general anesthesia are often associated with significant hemodynamic changes (hypotension and rapid blood pressure fluctuations) that could exacerbate ischemic injury. ${ }^{14}$ It is possible that the poor autoregulation and higher rates of intraprocedural hypotension with general anesthesia contribute to the markedly lower recanalization rates compared with conscious sedation seen in this meta-analysis.

General anesthesia remains widely used for intra-arterial treatment of acute ischemic stroke. The main argument in favor of general anesthesia is decreased intraprocedural patient movement. ${ }^{5}$ Patients who are awake during endovascular treatment may move during endovascular therapy, which could compromise the safety and efficacy of the intervention. ${ }^{15}$ Patient movement during the procedure can lead to wire perforation, resulting in intracranial hemorrhage or vascular injury in the form of dissection. However, no studies have demonstrated that conscious sedation is associated with higher rates of wire perforation, dissection, or intracranial hemorrhage than general anesthesia. ${ }^{16,17}$ Many practitioners cite perceived procedural safety, specifically avoiding emergent endotracheal intubation, as an advantage of general anesthesia over conscious sedation. ${ }^{3}$
Emergent intubation is associated with various complications including airway trauma, aspiration, and death. ${ }^{18,19}$ However, many studies have suggested that the rate of emergent endotracheal intubation among patients with conscious sedation receiving neuroendovascular therapies is very low. ${ }^{17,20-22}$ The findings of this meta-analysis that conscious sedation was actually associated with higher rates of recanalization and no increase in intraprocedural complications should assuage concerns about the perceived disadvantages of conscious sedation for the interventionalist.

In contrast to general anesthesia, conscious sedation allows intraprocedural monitoring of neurologic deficits, allowing operators to adjust the treatment strategy if needed. ${ }^{23}$ Dynamic cerebral autoregulation is preserved with midazolam sedation, resulting in decreased volatility in intraprocedural cerebral perfusion. ${ }^{24}$ While some studies have found that conscious sedation is associated with more rapid reperfusion, our study found no difference in procedure time, time to groin, or time from symptom onset to reperfusion between the general anesthesia and conscious sedation groups. ${ }^{25,26}$

Most previously published studies have demonstrated superior outcomes for patients undergoing endovascular recanalization therapy receiving conscious sedation relative to those receiving general anesthesia. General anesthesia is generally associated with higher rates of poor neurologic outcome at 90 days and higher mortality compared with conscious sedation. ${ }^{12,16,17,25,27}$ The use of general anesthesia was independently associated with worse outcomes despite comparable rates of recanalization among patients included in the North American SOLITAIRE Acute Stent Retriever Registry. ${ }^{28}$ One recently published study demonstrated that $>80 \%$ of patients undergoing mechanical thrombectomy could be safely treated while under conscious sedation, with high rates of good neurologic outcome. ${ }^{29}$ These studies, along with the findings from our study, suggest that conscious sedation is safe and effective in the setting of mechanical thrombectomy for acute ischemic stroke and should be preferred when deemed feasible.

\section{Limitations}

This study has several limitations. None of the available studies were randomized by anesthesia type. In general, average baseline NIHSS scores were higher for patients receiving general anesthesia than for those receiving conscious sedation. Adjustment for this variable was likely unreliable because of the small number of available studies with this information. Worse initial stroke severity could contribute to the higher rates of posttreatment morbidity and mortality seen in the general anesthesia group. However, the odds ratios found in this meta-analysis were not marginal, which strongly suggests that conscious sedation does lead to improved outcomes. Still, the higher baseline NIHSS scores underscore the possibility of selection bias because it is likely that patients with more severe strokes received general anesthesia or were intubated before the procedure due to an inability to preserve airway patency. We did not stratify outcomes on the basis of stroke location (anterior/posterior circulation) or initial ASPECTS.

The site of vascular occlusion is another determinant of outcomes and a potential confounder in this analysis. For example, carotid occlusions, carotid bifurcation occlusions, and basilar ar- 
tery occlusions typically have worse outcomes. It is possible that the lower recanalization rates with general anesthesia seen in this meta-analysis were the result of a higher proportion of more challenging vascular occlusions in the general anesthesia group. Although some of the studies included in our meta-analysis represented earlier experience with endovascular stroke therapy and may have lower rates of recanalization and higher risk of complications than those in current practice, this caveat should apply similarly to both anesthesia types. We were not able to evaluate the presence of publication bias because our study incorporated 9 studies and measures of publication bias are only reliable if $\geq 10$ studies are included in a meta-analysis.

\section{CONCLUSIONS}

This systematic review and meta-analysis demonstrates that patients with acute stroke receiving conscious sedation had significantly lower rates of morbidity and mortality and higher recanalization rates compared with patients receiving general anesthesia. However, these findings are based solely on studies that did not randomize patients by anesthesia type; differences in baseline patient characteristics, such as stroke severity, may confound the association. Therefore, a randomized controlled trial is needed to confirm whether general anesthesia is associated with higher rates of complications and impaired neurologic outcome in patients receiving endovascular therapy for acute ischemic stroke. In the meantime, when possible, it is reasonable to favor conscious sedation over general anesthesia during acute endovascular stroke therapy on the basis of available collective evidence.

Disclosures: Giuseppe Lanzino_UNRELATED: Consultancy: Covidien, ${ }^{\star}$ Codman/ Johnson \& Johnson,* Edge Therapeutics.* David F. Kallmes—UNRELATED: Board Membership: GE Healthcare (advisory board); Consultancy: ev3, ${ }^{*}$ Comments: planning and implementing clinical trials; Grants/Grants Pending: ev3,* MicroVention,* Sequent Medical, ${ }^{*}$ Surmodics, ${ }^{*}$ Codman/Johnson \& Johnson, ${ }^{*}$ Comments: preclinical and clinical research; Royalties: University of Virginia Patent Foundation (spine fusion). *Money paid to the institution.

\section{REFERENCES}

1. Brinjikji W, Rabinstein AA, Kallmes DF, et al. Patient outcomes with endovascular embolectomy therapy for acute ischemic stroke: a study of the National Inpatient Sample: 2006 to 2008. Stroke 2011; 42:1648-52

2. Saver JL. Time is brain-quantified. Stroke 2006;37:263-66

3. McDonagh DL, Olson DM, Kalia JS, et al. Anesthesia and sedation practices among neurointerventionalists during acute ischemic stroke endovascular therapy. Front Neurol 2010;1:118

4. Molina CA, Selim MH. General or local anesthesia during endovascular procedures: sailing quiet in the darkness or fast under a daylight storm. Stroke 2010;41:2720-21

5. Brekenfeld C, Mattle HP, Schroth G. General is better than local anesthesia during endovascular procedures. Stroke 2010;41:2716-17

6. Gupta R. Local is better than general anesthesia during endovascular acute stroke interventions. Stroke 2010;41:2718-19

7. Deeks JJ, Dinnes J, D'Amico R, et al. Evaluating non-randomised intervention studies. Health Technol Assess 2003;7:iii-x, 1-173

8. DerSimonian R, Laird N. Meta-analysis in clinical trials. Control Clin Trials 1986;7:177-88

9. Higgins JP, Thompson SG, Deeks JJ, et al. Measuring inconsistency in meta-analyses. BMJ 2003;327:557-60

10. Messick JM Jr, Newberg LA, Nugent M, et al. Principles of neuroan- esthesia for the nonneurosurgical patient with CNS pathophysiology. Anesth Analg 1985;64:143-74

11. Steen PA. Inhalational versus intravenous anesthesia: cerebral effects. Acta Anaesthesiol Scand Suppl 1982;75:32-35

12. Davis MJ, Menon BK, Baghirzada LB, et al. Anesthetic management and outcome in patients during endovascular therapy for acute stroke. Anesthesiology 2012;116:396-405

13. Petersen KD, Landsfeldt U, Cold GE, et al. Intracranial pressure and cerebral hemodynamic in patients with cerebral tumors: a randomized prospective study of patients subjected to craniotomy in propofol-fentanyl, isoflurane-fentanyl, or sevoflurane-fentanyl anesthesia. Anesthesiology 2003;98:329-36

14. Rosenberg M, Weaver J. General anesthesia. Anesth Prog 1991;38: $172-86$

15. Rossitti S, Pfister M. 3D road-mapping in the endovascular treatment of cerebral aneurysms and arteriovenous malformations. Interv Neuroradiol 2009;15:283-90

16. Abou-Chebl A, Lin R, Hussain MS, et al. Conscious sedation versus general anesthesia during endovascular therapy for acute anterior circulation stroke: preliminary results from a retrospective, multicenter study. Stroke 2010;41:1175-79

17. Jumaa MA, Zhang F, Ruiz-Ares G, et al. Comparison of safety and clinical and radiographic outcomes in endovascular acute stroke therapy for proximal middle cerebral artery occlusion with intubation and general anesthesia versus the nonintubated state. Stroke 2010;41:1180-84

18. Jabre P, Avenel A, Combes X, et al. Morbidity related to emergency endotracheal intubation: a substudy of the KETAmine SEDation trial. Resuscitation 2011;82:517-22

19. Li J, Murphy-Lavoie H, Bugas C, et al. Complications of emergency intubation with and without paralysis. Am J Emerg Med 1999; 17:141-43

20. Chamczuk AJ, Ogilvy CS, Snyder KV, et al. Elective stenting for intracranial stenosis under conscious sedation. Neurosurgery 2010;67: 1189-93; discussion 1194

21. Hassan AE, Chaudhry SA, Zacharatos $\mathrm{H}$, et al. Increased rate of aspiration pneumonia and poor discharge outcome among acute ischemic stroke patients following intubation for endovascular treatment. Neurocrit Care 2012;16:246-50

22. Qureshi AI, Suri MF, Khan J, et al. Endovascular treatment of intracranial aneurysms by using Guglielmi detachable coils in awake patients: safety and feasibility. J Neurosurg 2001;94:880-85

23. Abou-Chebl A, Krieger DW, Bajzer CT, et al. Intracranial angioplasty and stenting in the awake patient. J Neuroimaging 2006; 16:216-23

24. Ogawa Y, Iwasaki K, Aoki K, et al. The different effects of midazolam and propofol sedation on dynamic cerebral autoregulation. Anesth Analg 2010;111:1279-84

25. Li F, Deshaies EM, Singla A, et al. Impact of anesthesia on mortality during endovascular clot removal for acute ischemic stroke. $\mathrm{J} \mathrm{Neu-}$ rosurg Anesthesiol 2014;26:286-90

26. Langner S, Khaw AV, Fretwurst T, et al. Endovascular treatment of acute ischemic stroke under conscious sedation compared to general anesthesia: safety, feasibility and clinical and radiological outcome [in German]. Rofo 2013;185:320-27

27. Nichols $\mathrm{C}$, Carrozzella J, Yeatts $\mathrm{S}$, et al. Is periprocedural sedation during acute stroke therapy associated with poorer functional outcomes? J Neurointerv Surg 2010;2:67-70

28. Abou-Chebl A, Zaidat OO, Castonguay AC, et al. North American SOLITAIRE Stent-Retriever Acute Stroke Registry: choice of anesthesia and outcomes. Stroke 2014;45:1396-401

29. Soize S, Kadziolka K, Estrade L, et al. Mechanical thrombectomy in acute stroke: prospective pilot trial of the Solitaire FR device while under conscious sedation. AJNR Am J Neuroradiol 2013;34:360-65

30. Sugg RM, Jackson AS, Holloway W, et al. Is mechanical embolectomy performed in nonanesthetized patients effective? AJNR Am J Neuroradiol 2010;31:1533-35 